\title{
Synthesis and Antiproliferative Activities of 1-Substituted-3-(3-chloro-5- methoxyphenyl)-4-pyridinylpyrazole Derivatives Against Melanoma Cell Line
}

\author{
Won-Kyoung Choi and Chang-Hyun $\mathrm{Oh}^{-}$ \\ Biomaterials Research Center, Korea Institute of Science and Technologv, Seoul 130-650. Korea \\ ${ }^{\star} E$-mail: chohtakist.re.kr \\ Received July 16. 2009, Accepted July 30. 2009
}

\begin{abstract}
The synthesis of a new series of diarylureas and amides having a l-substituted-3-(3-chloro-5-methoxyphenyl)-4pyridiny lpyrazole scaffold is reported here. The in vitro antiproliferative activities of these diaryl derivatives against human melanoma cell line A375 were tested and the effect of substituents on the phenyl ring was investigated. Most of the newly synthesized compounds generally showed superior or similiar activity against A375 to Sorafenib. Among these compounds, Id, Ing and Ill showed excellent activity against A375 compared to Sorafenib.
\end{abstract}

Key Words: 4-Pyridinylpyrazole, A375, Antiproliferative activity. Melanoma

\section{Introduction}

Melanoma, the most aggressive form of skin cancer. is the fastest growing cancer in the United States. ${ }^{1, ?}$ Early stage melanoma can be cured surgically. However melanoma metastasizing to major organs (stage IV) is virtually incurable. Patients with disseminated melanoma have a median survival time of less than a year, and the estimated 5-years survival rate is less than $15 \%{ }^{-{ }^{2}}$ With the rapid incidence of melanoma in the United States and other developed countries. there is an urgent need to develop more effective drugs. ${ }^{t .6}$

Recently. Sorafenib. as well as other diarylureas. have been evaluated as potent and selective antiproliferative agents for the treatment of melanoma. ${ }^{i-1}$. The promising results have encouraged many research groups to investigate diarylurea scaffolds to develop new derivatives for the treatment of cancer. ${ }^{13-1 ?}$ Accordingly' we have stynthesized a new series of diaryl ureas. and reported their antiproliferative activity against A375 human melanoma cell line

In this work, we investigated the effect of replacing the 2-(methy laminocarbonyl)py ridine-4-yloxy group in Sorafenib with a 3-(3-chloro-5-methoxyphenyl)-4-pyridinyl-pyrazole moiety on the antiproliferative activity. The effect of substitution at the other phenylurea ring is also investigated by using the different substituents $\mathrm{R}$.

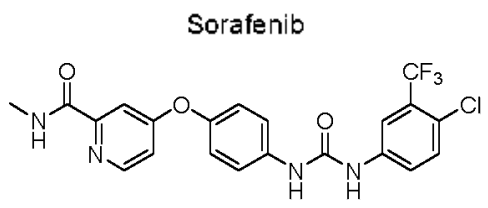

1-Substituted-3-(3-chloro-5-methoxyphenyl)-4-pyridinyl-pyrazole<smiles>COc1cc(Cl)cc(-c2nn(-c3ccc(NC(=O)P)cc3)cc2-c2ccncc2)c1</smiles>

\section{Results and Discussion}

Chemistry. 1.3.4-Triary lpy razole derivatives (Ia-f and IIa-e) with urea moiety at the terninal part were prepared according to the sequence of reactions shown in Scheme 1. Heating 3.5-dichlorobenzoic acid (1) with two molecular equivalents of sodium methoxide in hexamethylphosphoramide (HMPA) gave 3-chloro-5-methoxybenzoic acid (2). which upon esterification with methanol in the presence of acetyl chloride afforded the corresponding methyl ester 3 . The pyridyl derivative $\mathbf{4}$ was obtained by treatment of $\mathbf{3}$ with 4-picoline in THF in the presence of lithium bis(trimethylsilyl)amide (LHMDS). 3.4Diarylpyrazole derivative 5 was obtained by treatment of 4 with dimethylformamide dimethyl acetal (DMF-DMA), and subsequent treatment with lrydrazine monohydrate. 1,3,4-Triarylpy razole derivative 7 with amino group was prepared through N-arylation of 5 using 1-iodo-4-nitrobenzene in DMSO in the presence of anhydrous $\mathrm{K}_{2} \mathrm{CO}_{3}$. and subsequent reduction of the nitro group of 6 using stannous chloride. Interaction of the amino group with phenylisocyanate derivatives in THF afforded the corresponding urea derivatives (Ia-f). Demethylation of the methosy group of compounds Ia-f using $\mathrm{BBr}_{3}$ afforded the corresponding hydrosy derivatives (IIa-e).

The synthesis of Ig-i and Iff-h with amide moiety at the terminal part was carried out by the same procedure described for synthesis of urea derivatives (Ia-f and Ia-e). Benzoic acid derivatives were used instead of phenylisocyanate derivatives for acylation of amino group of 7 in DMF in the presence of HOBt and EDCI to afford Ig-i. Demethy lation of the methoxy group in order to obtain the corresponding hydroxy derivatives (IIf-h) was achieved by the same method described for preparation of IIta-e.

In vitro activity. The antiproliferative activity of the newly synthesized compounds against A375 human melanoma cell line was examined. The ability of these compounds to inhibit the growth of A375 cell line is summarized in Tables 1 and 2 . Sorafenib was selected as a reference standard. because it has been extensively used in clinical trials for melanoma. ${ }^{+18}$ Regarding the terminal substituents on the phenyl ring of the 
<smiles>CC(=O)c1cc(Cl)cc(Cl)c1</smiles><smiles>[CH-]=C</smiles><smiles>COc1cc(Cl)cc(-c2n[nH]cc2-c2ccncc2)c1</smiles><smiles>[R]NC(=O)Nc1ccc(-n2cc(-c3ccncc3)c(-c3cc(Cl)cc(OC)c3)n2)cc1</smiles>

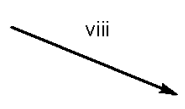

la If<smiles>COc1cc(Cl)cc(C(=O)O)c1</smiles>

2<smiles>C=C</smiles><smiles>COc1cc(Cl)cc(-c2nn(-c3ccc([N+](=O)[O-])cc3)cc2-c2ccncc2)c1</smiles>

6<smiles>[Z]NC(=O)Nc1ccc(-n2cc(-c3ccncc3)c(-c3cc(O)cc(Cl)c3)n2)cc1</smiles>

Ila Ile

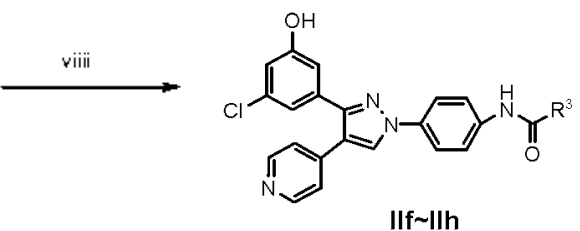

Scheme 1. Reagents and conditions: i) sodium methoxide, HMPA; ii) acetyl chloride, MeOH: iii) 4-picoline, LHMDS, THF; iv) (a) DMF-DMA (b) hydrazine monohydrate, $\mathrm{EtOH}$ y) 1-iodo-4-nitrobenzene, $\mathrm{K}_{2} \mathrm{CO}_{3}$, CuI, L-proline, DMSO; vi) Raney nickel, 1,4-dioxane; vii) $\mathrm{R}^{2} \mathrm{NCO}$, THF; viii) $\mathrm{R}^{3} \mathrm{COOH}, \mathrm{HOBt}, \mathrm{EDCI}, \mathrm{TEA}, \mathrm{DMF}$; viiii) $\mathrm{BBr}_{3} \mathrm{CH}_{2} \mathrm{Cl}_{2}$.

tail. it was found that compounds Ih. If. IIg, and Ih having amide moiety were more potent than compounds $\mathbf{~ b}$, II $\mathbf{~ I l b}$. and Ic having urea moiety. As to the substituents on the chlorophenyl ring, compounds Ilb. IId, Ile, If, IIg. and Ih with hydroxyl group showed higher antiproliferative activity in comparison with the corresponding methosy derivatives $\mathbf{I b}$. Id. Ie, Ig. Ih. and Ii. This indicates that the more polar substituent at this position is preferable. Most of the newly synthesized compounds generally showed superior or similiar activity against A375 to Sorafenib. Among these compounds. IId. II and Ih showed excellent activity against A375 compared to Sorafenib.

\section{Experimental Section}

Melting points (mp) were determined on a Walden Precision Apparatus Electrothermal 9300 apparatus and were uncorrected. ${ }^{\mathrm{H}} \mathrm{H}-\mathrm{NMR}$ spectral data were recorded using a Gemini 300 spectrometer. Mass spectra were recorded on a Waters 3100 Mass Detecter.

A375P Cell culture and anti-proliferative activity of tested compound on A375P. A375P cells were purchased from American Type Culture Collection (ATCC, Rockville, MD, US) and maintained in a DMEM (Welgene. Daegu. Korea) supplemented with $10 \%$ FBS (Welgene) and $1 \%$ penicillin/streptomycin (Welene) in a humidified atmosphere with $5 \% \mathrm{CO}_{2}$ at $37^{\circ} \mathrm{C}$. A375P cells were taken from culture substrate with $0.05 \%$ trypsin- $0.02 \%$ EDTA and plated at a density of $5 \times 10^{3}$ cells $/$ well in 96 well plates and then incubated at $37^{\circ} \mathrm{C}$ for 24 hours in a humidified atmosphere with $5 \% \mathrm{CO}_{2}$ prior to treatment with various concentrations (three fold serial dilution. 12 points) of test compounds. The A375P cell viability was assessed by the conventional 3-(4.5-dimethylthiazol-2-yl)-2.5-diphenyltetrazolium bronide (MTT) reduction assay. MTT assays were carried out with CellTiter 96 (Promega) according to the manufacturer's instructions. The absorbance at $590 \mathrm{~mm}$ was recorded using EnVision2103 (PerkinElmer; Boston. MA. US). The $\mathrm{IC}_{51}$ was calculated using GraphPad Prism 4.0 softwere.

3-Chlo10-5-methoxybenzoic acid (2). A mixture of 3.5-dichlorobenzoic acid (1. $1.0 \mathrm{~g}, 5.2 \mathrm{~nm}$ ol) and NaOMe $(3.5 \mathrm{~mL}$, $25 \mathrm{wt} \%$ solution in methanol. $16.2 \mathrm{nmol})$ in $\operatorname{HMPA}(2.0 \mathrm{~mL})$ was heated at $120^{\circ} \mathrm{C}$ for $15 \mathrm{~h}$. The mixture was poured into ice-water and acidified with conc. $\mathrm{HCl}$ to give 3-chloro-5methoxybenzoic acid $(2,1.2 \mathrm{~g} .80 \%)$ as a precipitate which was collected by filtration and used in the next step without purification. ${ }^{\mathrm{l}} \mathrm{H}-\mathrm{NMR}$ (DMSO- $d_{6,} 300 \mathrm{MHz}$ ) $\delta$ 7.47 (t. $\mathrm{lH}, J=$ $1.6 \mathrm{~Hz}) .7 .38(\mathrm{q} . \mathrm{lH}, J=1.3 \mathrm{~Hz}) .7 .30(\mathrm{t} .1 \mathrm{H}, J=2.0 \mathrm{~Hz}) .3 .82$ (s. $3 \mathrm{H})$.

Methyl 3-chlo10-5-methoxybenzoate (3). Acetyl clloride $(1.9 \mathrm{~nL}, 28.1 \mathrm{mmol})$ was added dropwise to a solution of $(2$, $1.0 \mathrm{~g} .5 .4 \mathrm{mmol}$ in $\mathrm{MeOH}(40.0 \mathrm{~mL})$ at $0^{\circ} \mathrm{C}$ and the reaction nixture was then stirred at room temperature for $15 \mathrm{lh}$. After evaporation of the organic solvent, the residue was purified by flash column chromatography (silica gel. hexanes-ethyl acetate $5: 1 \mathrm{v} / \mathrm{v})$ to give $(3,1.7 \mathrm{~g}, 65 \%)$ as an oil. ${ }^{\mathrm{l}} \mathrm{H}-\mathrm{NMR}\left(\mathrm{CDCl}_{3}\right.$, 
Table 1. Antiproliferative activity of the urea substituted compounds<smiles>[R]NC(=O)Nc1ccc(-n2cc(-c3ccncc3)c(-c3cc(Cl)cc(O[R])c3)n2)cc1</smiles>

Comd.

$300 \mathrm{MHz})$ ò $7.61(\mathrm{t}, 1 \mathrm{H} . J=1.6 \mathrm{~Hz}), 7.45(\mathrm{q}, 1 \mathrm{H}, J=1.3 \mathrm{~Hz})$, 7.09 (t. IH. $J=2.2 \mathrm{~Hz}$ ) 3.92 (s. $3 \mathrm{H}), 3.85$ (s. $3 \mathrm{H}$ ).

1-(3-Chlon-5-methoxy phenyl)-2-(py ridin-t-yl)ethanone (4). To a mixture of compound (3. $1.0 \mathrm{~g} .5 .0 \mathrm{mmol})$ and 4-picoline $(0.5 \mathrm{~mL}, 5.6 \mathrm{mmol})$ in THF $(5.0 \mathrm{~mL})$ in a water bath at $-20^{\circ} \mathrm{C}$. LHMDS $(3.7 \mathrm{~mL}, 1.0 \mathrm{M}$ solution in THF, 19.9 mmol) was slowly added to maintain the temperature at -20
Table 2. Antiproliferative activity of the amide substituted compounds

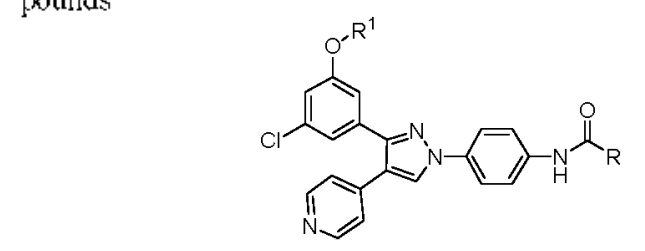

\begin{tabular}{|c|c|c|c|}
\hline Comd. & $\mathrm{R}^{1}$ & $\mathrm{R}$ & $\mathrm{A} 375 \mathrm{P}\left(\mathrm{IC}_{3(1,-4 \mathrm{M}}\right)$ \\
\hline go & $\mathrm{CH}_{3}$ & & $>20$ \\
\hline Ih & $\mathrm{CH}_{3}$ & & 2.5 \\
\hline Ii & $\mathrm{CH}_{3}$ & & $>20$ \\
\hline IIf & $\mathrm{H}$ & & 2.2 \\
\hline IIg & $\mathrm{H}$ & & 1.7 \\
\hline Ilh & $\mathrm{H}$ & & 0.9 \\
\hline Sorafenib & & & 5.0 \\
\hline
\end{tabular}

${ }^{\circ} \mathrm{C}$. The reaction mixture was stirred overnight at room temperature. The mixture was quenched with saturated aqueous $\mathrm{NH}_{4} \mathrm{Cl}$. Ethyl acetate was added and the organic layer was separated. The aqueous layer was extracted with ethyl acetate. The combined organic layer extracts were washed with brine and dried over $\mathrm{Na}_{2} \mathrm{SO}_{4}$. The organic solvent was evaporated under reduced pressure and the residue was purified by flash column chromatography' (silica gel, hexanes-ethyl acetate $1: 1$ $\mathrm{v} / \mathrm{v}$ then switching to hexanes-ethyl acetate $1.5 \mathrm{v} / \mathrm{v})$ to yield compound (t, $3.3 \mathrm{~g}, 40 \%)$. ${ }^{\mathrm{H}} \mathrm{H}-\mathrm{NMR}\left(\mathrm{CDCl}_{3} .300 \mathrm{MHz}\right) \hat{o}$ 8.58 (d. $2 \mathrm{H}, J=5.7 \mathrm{~Hz}) .7 .54$ (t. $1 \mathrm{H} . J=1.5 \mathrm{~Hz}) .7 .39$ (q. $1 \mathrm{H}$. $J=1.4 \mathrm{~Hz}) .7 .19(\mathrm{~d}, 2 \mathrm{H} . J=5.7 \mathrm{~Hz}) .7 .12(\mathrm{t} .1 \mathrm{H}, J=2.0 \mathrm{~Hz})$, 4.25 (s. $2 \mathrm{H}) .3 .85$ (s. $3 \mathrm{H})$

4-[3-(3-Chloro-5-methoxyphenyl)-1 $H$-pyrazol-4-y]]pyridime (5). Compound $(4,1.0 \mathrm{~g}, 3.8 \mathrm{~mm}$ ) $)$ was added to DMF-DMA $(5.14 \mathrm{~mL}, 38.2 \mathrm{mmol}$ ) and the mixture was stirred at room temperature for $18 \mathrm{~h}$. The resulting solution was concentrated to dnyness to furnish an oil which was used in the next step without any purification. To a portion of the oil from the previous step $(0.137 \mathrm{~g} .0 .457 \mathrm{mmol}) \mathrm{in} \mathrm{EtOH}(3.0 \mathrm{~mL})$ was added hydrazine monohydrate $(0.04 \mathrm{~mL}, 0.76 \mathrm{mmol})$ and the reaction was stirred overnight at room temperature. Water $(5.0 \mathrm{~mL})$ was added to the reaction mixture and the organics were extracted with ethyl acetate. The conbined organic layer extracts were washed 
with brine and dried over $\mathrm{MgSO}_{4}$. After evaporation of the organic solvent. the residue was purified by column chromatography (silica gel. hexanes-ethyl acetate $1: 1 \mathrm{v} / \mathrm{v}$ then switching to hexanes-ethyl acetate $1: 5 \mathrm{~F} / \mathrm{v})$ to yield compound (5. $0.11 \mathrm{~g} .81 \%)$. ${ }^{\mathrm{H}} \mathrm{H}-\mathrm{NMR}\left(\mathrm{CDCl}_{3 .} 300 \mathrm{MHz}\right) \delta 8.55$ (d. $2 \mathrm{H} . J=$ $5.3 \mathrm{~Hz}), 7.83$ (s. $1 \mathrm{H}), 7.23(\mathrm{~d}, 2 \mathrm{H}, J=5.4 \mathrm{~Hz}) .7 .06(\mathrm{brs}, 1 \mathrm{H})$, 6.94 (brs $1 \mathrm{H}$ ) 6.85 (brs. $\mathrm{lH}$ ). 3.77 (s. $3 \mathrm{H}$ ).

4-[3-(3-Chloro-5-methoxy-phenyl)-1-(4-nitrophenyl)-1 $\mathrm{H}$ py razol-4-yl]pyridine (6). A mixture of compound $(5,0.5 \mathrm{~g}$. $1.7 \mathrm{mmol}) .1$-iodo-4-nitrobenzene $(0.9 \mathrm{~g} .3 .5 \mathrm{mmol}) . \mathrm{K}_{2} \mathrm{CO}_{3}$ $(0.7 \mathrm{~g}, 5.2 \mathrm{mmol}), \mathrm{CuI}(0.033 \mathrm{~g}, 0.2 \mathrm{mmol})$. and L-proline $(0.0+\mathrm{g} .0 .2 \mathrm{mmol})$ in DMSO $(7.0 \mathrm{~mL})$ was heated at $90^{\circ} \mathrm{C}$ under nitrogen atmosphere for $8 \mathrm{~h}$. The cooled solution was partitioned between water and ethyl acetate. The organic phase was washed with brine ( 3 times) and dried over $\mathrm{Na}_{2} \mathrm{SO}_{4}$. After evaporation of the organic solvent, the residue was purified by column chromatography (silica gel, hexanes-ethyl acetate 1:5 $\checkmark / \mathrm{s})$ to yield compound $(6.0 .8 \mathrm{~g}, 84 \%)$. ${ }^{\mathrm{l}} \mathrm{H}-\mathrm{NMR}$ (DMSO- $d_{6}$. $300 \mathrm{MHz}$ ) ô 9.25 (s. $1 \mathrm{H}$ ) 8.44 (d. $2 \mathrm{H} . J=9.5 \mathrm{~Hz}$ ) 8.25 (d. $2 \mathrm{H}$. $J=8.5 \mathrm{~Hz}), 8.06(\mathrm{~d}, 2 \mathrm{H} . J=8.7 \mathrm{~Hz}) .7 .97($ d. $2 \mathrm{H} . J=8.0 \mathrm{~Hz})$, 7.41 (brs, 2H), 6.98 (brs. $1 \mathrm{H}) .3 .74$ (s, 3H).

4-[3-(3-Chloro-5-methoxyphenyl)-1-(4-aminophenyl)-1 Hpyrazol-4-yl]pyridine (7). A mixture of compound $(6,0.5 \mathrm{~g}$. $1.2 \mathrm{mmol}$ ) and Raney nickel $(1.0 \mathrm{~g})$ in l.t-dioxane $(5.0 \mathrm{~mL}$ ) was stirred at room temperature under hydrogen atmosphere for $6 \mathrm{~h}$. The mixture was filtered through celite and the filtrate was evaporated under reduced pressure to give compound ( 7 . $0.5 \mathrm{~g}, 87 \%)$. ${ }^{\mathrm{l}} \mathrm{H}-\mathrm{NMR}\left(\mathrm{CDCl}_{3}, 300 \mathrm{MHz}\right)$ ò 8.55 (d, $2 \mathrm{H} . J=$ $4.6 \mathrm{~Hz}$ ). 7.98 (s. IH). 7.53 (d. $2 \mathrm{H}, J=8.7 \mathrm{~Hz}$ ). 7.25 (d. $2 \mathrm{H} . J=$ $5.2 \mathrm{~Hz}$ ). 7.19 (brs. $1 \mathrm{H}), 6.93$ (brs, $1 \mathrm{H}), 6.92$ (brs, $1 \mathrm{H}), 6.77$ (d, $2 \mathrm{H} . J=8.7 \mathrm{~Hz}$ ) 3.73 (s. $\mathrm{lH}$ ).

1-\{t-[3-(3-Chlono-5-methoxy]heny])-4-pyridin-t-y]-pyrazol1-yl]-phenyl\} -3-(3,4-dichlomphenyl)urea (Ia). A solution of compound ( $7.50 \mathrm{mg} .0 .1 \mathrm{mmol})$ in THF $(1.0 \mathrm{~mL})$ was treated with dropwise addition of a solution of 3,4-dichlorophenyl isocyanate ( $25.0 \mathrm{mg} .0 .1 \mathrm{mmol})$ in THF $(1.0 \mathrm{~mL})$ at room temperature under $\mathrm{N}_{2}$ and the mixture was stirred at room temperature for $12 \mathrm{~h}$. The mixture was evaporated under reduced pressure. and the residue was purified by column chromatography (silica gel. hexanes-ethyl acetate $1: 5 \mathrm{~F} /$ ) to yield compound Ia (18.0 mg $24 \%$ ). mp: $173-175^{\circ} \mathrm{C}$. (dec.). ${ }^{1} \mathrm{H}-\mathrm{NMR}$ $\left(\mathrm{CDCl}_{3} .300 \mathrm{MHz}\right) \delta 8.56$ (d. $\left.2 \mathrm{H} . J=6.0 \mathrm{~Hz}\right), 7.93$ (brs. $1 \mathrm{H}$ ). 7.86 (d. $2 \mathrm{H} . J=8.5 \mathrm{~Hz}) .7 .65$ (d. $2 \mathrm{H} . J=8.4 \mathrm{~Hz}) .7 .5 \mathrm{l}(\mathrm{s} . \mathrm{HH})$. 7.36 (brs, 4H). 7.13 (s. 1H). 7.10 (s. $1 \mathrm{H}), 6.96(\mathrm{~s}, 1 \mathrm{H}), 7.75$ (s, $3 \mathrm{H})$. ESI-MS: $564.0[\mathrm{M}+\mathrm{H}]^{-}$.

The synthesis of compounds Ib-If were canied out by the same procedure as described for the preparation of Ia

Ib: Yield $23 \%$. mp: $195-197^{\circ} \mathrm{C}$. (dec.). ${ }^{\mathrm{l}} \mathrm{H}-\mathrm{NMR}\left(\mathrm{CDCl}_{3}\right.$. $300 \mathrm{MHz}$ ) ò 8.58 (brs, 2H), 8.10 (s. $1 \mathrm{H}), 7.80$ (s. 1H). 7.77 (s, IH). 7.73 (s. IH). $7.6 \mathrm{l}$ (s. IH). $7.5+$ (d. $2 \mathrm{H}, J=8.9 \mathrm{~Hz}) .7 .46$ (brs. $2 \mathrm{H}$ ) 6.92 (brs. $2 \mathrm{H}$ ) 6.68 (s. IH). 6.62 (s. IH) $3.7+$ (s. $3 \mathrm{H})$. ESI-MS: $598.0[\mathrm{M}+\mathrm{H}]^{+}$

Ic: Yield $29 \%$. ${ }^{1} \mathrm{H}-\mathrm{NMR}\left(\mathrm{CDCl}_{3} .300 \mathrm{MHz}\right) \delta 8.58$ (brs. $2 \mathrm{H}), 8.08(\mathrm{~s}, 1 \mathrm{H}) .7 .94$ (bIs. $\mathrm{lH}), 7.73(\mathrm{~d}, 2 \mathrm{H} . J=8.9 \mathrm{~Hz}) .7 .56$ (s. IH). 7.53 (brs. $2 \mathrm{H}$ ) .7 .36 (s. $3 \mathrm{H}$ ) .7 .19 (brs. IH). 6.92 (brs. 2H). $3.7+$ (s. $3 \mathrm{H})$. ESI-MS: $632.0[\mathrm{M}+\mathrm{H}]^{-}$.

Id: Yield $35 \%$. mp: $222-224^{\circ} \mathrm{C}$. (dec.). ${ }^{1} \mathrm{H}-\mathrm{NMR}\left(\mathrm{CDCl}_{3}\right.$, $300 \mathrm{MHz}$ ) ò 9.78 (brs. IH) 8.94 (s. IH). $8.6 \mathrm{l}$ (brs. IH). 8.56 (d. $2 \mathrm{H} . J=6.1 \mathrm{~Hz}$ ). 7.89 (d. $2 \mathrm{H} . J=8.9 \mathrm{~Hz}$ ). 7.64 (d. $2 \mathrm{H} . J=$ $9.0 \mathrm{~Hz}) .7 .36(\mathrm{~d} .2 \mathrm{H} . J=6.2 \mathrm{~Hz}) .7 .32(\mathrm{~s}, 1 \mathrm{H}) .7 .13$ (brs. $\mathrm{lH}$ ). 7.09 (brs. $1 \mathrm{H}$ ). 6.96 (brs. IH). 3.74 (s. 3H). ESI-MS: 564.0 $\mathrm{M}+\mathrm{H}]$

Ie: Yield 43\%. mp: $237-239^{\circ} \mathrm{C}$. (dec.). ${ }^{\mathrm{l}} \mathrm{H}-\mathrm{NMR}\left(\mathrm{CDCl}_{3}\right.$. $300 \mathrm{MHz}) \delta 8.54$ (brs. $2 \mathrm{H}), 7.96(\mathrm{~s}, 1 \mathrm{H}) .7 .61$ (d, $2 \mathrm{H}, J=8.5$ Hz). 7.54 (s. IH). 7.40 (s. IH). 7.36 (s. IH). 7.26 (s. IH). $7.2 \mathrm{I}$ (d. $2 \mathrm{H}, J=4.4 \mathrm{~Hz}$ ). 7.15 (d. $2 \mathrm{H}, J=5.2 \mathrm{~Hz}$ ). 6.99 (brs, $1 \mathrm{H}$ ), 6.88 (brs. $2 \mathrm{H}$ ). 3.70 (s. $3 \mathrm{H}$ ). ESI-MS: $530.1[\mathrm{M}+\mathrm{H}]$

If: Yield $40 \%$. ${ }^{\mathrm{l}} \mathrm{H}-\mathrm{NMR}\left(\mathrm{CDCl}_{2 .} .300 \mathrm{MHz}\right) \delta 8.93$ (s. $\left.\mathrm{lH}\right)$. 8.56 (d. $2 \mathrm{H}, J=5.7 \mathrm{~Hz}$ ). 8.50 (s. $1 \mathrm{H}$ ). 7.91 (s. $1 \mathrm{H}$ ), 7.88 (s. $2 \mathrm{H}$ ). 7.64 (d. $2 \mathrm{H} . J=8.1 \mathrm{~Hz}$ ). 7.36 (d. $2 \mathrm{H}, J=5.9 \mathrm{~Hz}$ ). 7.12 (s. IH). $7.09(\mathrm{~s}, 1 \mathrm{H}) .6 .96(\mathrm{~s} .1 \mathrm{H}), 3.74(\mathrm{~s}, 3 \mathrm{H})$. ESI-MS: $598.0[\mathrm{M}+\mathrm{H}]^{-}$.

3,4-Dichloro- $N$-\{4-[3-(3-chloro-5-methoxy]henyl)-4-pyridin-4-yl-pyrazol-1-yl]phenyl\} benzamide (Ig). A mixture of compound (7. $50.0 \mathrm{mig}$. $0.1 \mathrm{~nm}$ nol), 3.4-dichlorophenyl benzoic acid (38.0 mg. $0.2 \mathrm{mmol}$ ). HOBt (36.0 $\mathrm{mg} .0 .3 \mathrm{mmol}$ ). and EDCI (38.0 mg, 0.2 nunol) in DMF (1.0 nL) was cooled to $0^{\circ} \mathrm{C}$ under nitrogen atmosphere. The mixture was warmed to room temperature and $\mathrm{Et}_{2} \mathrm{~N}(0.03 \mathrm{~mL}, 0.2 \mathrm{nmol})$ was added. The nuisture was stirred at $80^{\circ} \mathrm{C}$ for $12 \mathrm{~h}$. The reaction nixture was cooled and then partitioned between $\mathrm{H}_{2} \mathrm{O}$ and ethyl acetate. The organic layer was washed with brine and dried over $\mathrm{Na}_{2} \mathrm{SO}_{4}$. After evaporation of the organic solvent. the residue was purified by column cluromatography (silica gel, hexanes-ethyl acetate $1: 5 \mathrm{v} / \mathrm{v})$ to yield compound $\mathrm{Ig}(22.0 \mathrm{mg} .31 \%$ ). $\mathrm{mp}$ : $224-226^{\circ} \mathrm{C}$. (dec.). ${ }^{1} \mathrm{H}-\mathrm{NMR}$ (DMSO- $d_{6}, 300 \mathrm{MHz}$ ) ò 8.92 (s. $1 \mathrm{H}), 8.55(\mathrm{t}, 3 \mathrm{H}, J=4.8 \mathrm{~Hz}), 7.88($ d. $2 \mathrm{H} . J=9.0 \mathrm{~Hz}), 7.63$ (d. $2 \mathrm{H} . J=9.0 \mathrm{~Hz}$ ) $.7 .36(\mathrm{~d} .3 \mathrm{H}, J=5.8 \mathrm{~Hz}$ ). 7.32 (s. $\mathrm{lH}$ ). 6.97 (brs. 1H). 8.84 (brs, 2H) . ESI-MS: $550.8[\mathrm{M}+\mathrm{H}]^{+}$.

The synthesis of compounds $\mathrm{Ih}$ and $\mathrm{Ii}$ was canied out by the same procedure as described for the preparation of $\mathbf{~} \mathrm{g}$.

Ih: Yield $25 \%$. ${ }^{\mathrm{H}} \mathrm{H}-\mathrm{NMR}$ (DMSO- $\left.d s, 300 \mathrm{MHz}\right) \delta 8.93$ (s, lH). 8.56 (d. $2 \mathrm{H}, J=5.3 \mathrm{~Hz}$ ). 8.17 (brs. $2 \mathrm{H}$ ). 7.87 (d. $2 \mathrm{H} . J=$ $9.0 \mathrm{~Hz}$ ) 7.67 (brs, $3 \mathrm{H}$ ), 7.36 (d. $2 \mathrm{H}, J=6.0 \mathrm{~Hz}$ ), 6.97 (brs, lH). 6.84 (brs. $2 \mathrm{H}$ ). ESI-MS: $583.0[\mathrm{M}+\mathrm{H}]$

Ii: Yield 39\%. ${ }^{1} \mathrm{H}-\mathrm{NMR}$ (DMSO-ds. $300 \mathrm{MHz}$ ) ô $8.90(\mathrm{~s}$, lH). 8.55 (d. $2 \mathrm{H} . J=4.4 \mathrm{~Hz}$ ). 7.83 (d. $2 \mathrm{H} . J=8.9 \mathrm{~Hz}$ ). 7.75 (s. lH). 7.65 (d. $2 \mathrm{H}, J=8.3 \mathrm{~Hz}$ ) 7.36 (brs. $3 \mathrm{H}$ ). $7.0 \mathrm{l}$ (s. IH). 6.99 (s. $1 \mathrm{H}), 6.97$ (s, 1H). 6.83 (brs. $2 \mathrm{H}$ ). ESI-MS: $617.1[\mathrm{M}+\mathrm{H}]^{-}$

1-\{4-[3-(3-Chloro-5-methoxy-phenyl)-4-pyidin-4-y]-pylazol-1-yl]-phenyl;-3-(3,4-(lichlorophenyl)urea (IIa). To a mixture of compound $\mathbf{I a}(50.0 \mathrm{mg} .0 .1 \mathrm{mmol})$ in methylene chloride $(1.0 \mathrm{~mL}) . \mathrm{BBr}_{3}(0.02 \mathrm{~mL}$ of a $1 \mathrm{M}$ solution in $\mathrm{MC}, 0.3 \mathrm{numol})$ was added dropwise at $-78^{\circ} \mathrm{C}$ under $\mathrm{N}_{2}$ and the reaction mixture was stirred for $30 \mathrm{~min}$. The mixture was warmed to room temperature and stirred for $1 \mathrm{~h}$. The nuxture was quenched with saturated aqueous $\mathrm{NaHCO}_{3}$. Ethyl acetate was added and the orgauic layer was separated. The aqueous layer was extracted with ethyl acetate. The combined organic layer extracts were washed with brine. dried with $\mathrm{Na}_{2} \mathrm{SO}_{4}$. After evaporation of the organic solvent. the residue was purified by short column chromatography (silica gel, hexanes-ethyl acetate $1: 5 \mathrm{v} / \mathrm{v}$ ) to yield compound IIa $\left(45.0 \mathrm{mg} .92 \%\right.$ ). nip: $245-247^{\circ} \mathrm{C}$. (dec.). ${ }^{1} \mathrm{H}-\mathrm{NMR}$ (DMSO- $d_{0}, 300 \mathrm{MHz}$ ) ô 10.61 (s. $\mathrm{lH}$ ), 9.00 (s. lH), $8.57(\mathrm{~d}, 2 \mathrm{H} . J=4.9 \mathrm{~Hz}), 8.26(\mathrm{~s}, 1 \mathrm{H}), 7.96(\mathrm{~s}, 4 \mathrm{H}), 7.86(\mathrm{~d}, 1 \mathrm{H}$. $J=8.6 \mathrm{~Hz}), 7.37(\mathrm{~d} .2 \mathrm{H}, J=4.8 \mathrm{~Hz}), 7.1+(\mathrm{s}, 1 \mathrm{H}) .7 .11(\mathrm{~s} .1 \mathrm{H})$, 6.97 (s. $\mathrm{lH}) .3 .75$ (s. $3 \mathrm{H})$. ESI-MS: $550.0[\mathrm{M}+\mathrm{H}]^{+}$ 
The synthesis of compounds Illb-Illh was camied out by the same procedure as described for the preparation of IIa.

IIb: Yield 40\%. ${ }^{1} \mathrm{H}-\mathrm{NMR}$ (DMSO- $\tau_{6 .} .300 \mathrm{MHz}$ ) $\delta 10.71$ (s. 1H). 9.02 (brs, lH), 8.57 (brs. 2H) 8.42 (brs, $1 \mathrm{H}$ ), 8.27 (brs. lH). 7.96 (s. $4 \mathrm{H}$ ). 7.37 (brs. $2 \mathrm{H}$ ). 7.10 (s. $\mathrm{HH}) .7 .08(\mathrm{~s} . \mathrm{HH}$ ). $6.97(\mathrm{~s}, \mathrm{lH}) .3 .74(\mathrm{~s}, 3 \mathrm{H})$. ESI-MS: $584.0[\mathrm{M}+\mathrm{H}]^{+}$.

Ic: Yield $56 \%$ mp: $245-247^{\circ} \mathrm{C}$. (dec.). ${ }^{~} \mathrm{H}-\mathrm{NMR}$ (DMSO- $d_{6}$. $300 \mathrm{MHz}$ ) $\delta 8.59$ (d. $2 \mathrm{H} . J=4.9 \mathrm{~Hz}$ ), 8.38 (brs, $1 \mathrm{H}$ ). 8.18 (brs. $1 \mathrm{H}) .8 .14$ (brs. $1 \mathrm{H}$ ), 8.10 (brs, 1H). 7.85 (s. $3 \mathrm{H}) .7 .27$ (s. $3 \mathrm{H}$ ), 7.22 (brs. $1 \mathrm{H}$ ). 6.94 (brs. 2H). 3.75 (s. 3H). ESI-MS: 618.1 $[\mathrm{M}+\mathrm{H}]^{+}$

IId: Yield 87\% mp: $225-227^{\circ} \mathrm{C}$. (dec.). ${ }^{\mathrm{H}} \mathrm{H}-\mathrm{NMR}$ (DMSO- $t_{6}$ $300 \mathrm{MHz}) \delta 8.92(\mathrm{~s}, \mathrm{lH}), 8.55$ (d. $2 \mathrm{H}, J=4.8 \mathrm{~Hz}$ ). 8.17 (brs, lH) 7.85 (d. $2 \mathrm{H} . J=8.4 \mathrm{~Hz}$ ). 7.67 (s. $3 \mathrm{H}) .7 .64$ (brs. $\mathrm{lH}$ ). 7.35 (d. $2 \mathrm{H}, J=4.4 \mathrm{~Hz}$ ) 6.97 (brs. IH). 6.84 (brs. $2 \mathrm{H}$ ). ESI-MS: $550.0[\mathrm{M}+\mathrm{H}]^{-}$.

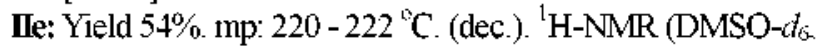
$300 \mathrm{MHz}) \delta 8.09$ (d. $2 \mathrm{H}, J=11.9 \mathrm{~Hz}) .8 .92(\mathrm{~s}, 1 \mathrm{H}) .8 .56$ (d. $2 \mathrm{H} . J=6.0 \mathrm{~Hz}) .7 .91(\mathrm{~s} . \mathrm{lH}) .7 .86(\mathrm{~d} . \mathrm{lH} . J=8.8 \mathrm{~Hz}) .7 .62(\mathrm{~d}$. $2 \mathrm{H} . J=8.9 \mathrm{~Hz}$ ). 7.35 (s. $3 \mathrm{H}$ ). 6.97 (brs, $1 \mathrm{H}$ ), 6.84 (brs. $2 \mathrm{H}$ ). ESI-MS: $516.0[\mathrm{M}+\mathrm{H}]^{-}$.

Iff: Yield $62 \%$ mp: $185-187^{\circ} \mathrm{C}$. (dec.). ${ }^{1} \mathrm{H}-\mathrm{NMR}$ (DMSO- $t_{6}$ $300 \mathrm{MHz}) \delta 2.26(\mathrm{~s}, 1 \mathrm{H}), 8.77$ (d, $2 \mathrm{H} . J=5.2 \mathrm{~Hz}), 8.26(\mathrm{~s} .1 \mathrm{H})$. 7.97 (s. $5 \mathrm{H}$ ). 7.86 (d. $1 \mathrm{H} . J=8.3 \mathrm{~Hz}$ ). 7.76 (d. $2 \mathrm{H} . J=5.4 \mathrm{~Hz}$ ). 7.03 (brs. 1H). 6.92 (brs, 1H). 6.86 (brs. lH). ESI-MS: 535.0 $[\mathrm{M}+\mathrm{H}]^{+}$.

IIg: Yield 61\% mp: $160-162{ }^{\circ} \mathrm{C} .{ }^{1} \mathrm{H}-\mathrm{NMR}$ (DMSO-d $d_{6 .} 300$ MHz) $\delta 8.99$ (s. 1H). 8.59 (brs, 2H). 8.43 (brs. 2H), 7.96 (s. $4 \mathrm{H}) .7 .52$ (s. IH). 7.39 (s. 2H), 6.99 (brs. IH). 6.88 (brs. lH). 6.86 (brs, lH). ESI-MS: $569.3[\mathrm{M}+\mathrm{H}]^{+}$.

Illi: Yield $72 \%$. mp: $240-242^{\circ} \mathrm{C}$. (dec.). ${ }^{~} \mathrm{H}-\mathrm{NMR}$ (DMSO-do. $300 \mathrm{MHz}$ ) $\delta 8.99$ (s. $1 \mathrm{H}$ ). $8.6+$ (brs. $2 \mathrm{H}$ ). 8.57 (d. $2 \mathrm{H} . J=6.1$ Hz). $8.41(\mathrm{~s}, 1 \mathrm{H}) .7 .97(\mathrm{~s} .4 \mathrm{H}), 7.37(\mathrm{~d}, 2 \mathrm{H} . J=6.0 \mathrm{~Hz}), 7.00$ (brs. $\mathrm{lH}$ ). 6.85 (brs. $\mathrm{lH}$ ). ESI-MS: $603.0[\mathrm{M}+\mathrm{H}]^{+}$.

\section{References}

1. Atallah, E.; Flaherty, L. Cmm Treat. Options Oncol 2005, 6, 185

2. Barth, A.; Wanek, L. A.; Morton, D. L. J. Am. Coll. Sung. 1995, $181,193$.

3. Anderson, C. M.; Buzaid, A. C.; Legha, S. S. Oncol . Williston Park) 1995, 9, 1149 .

4. Gray-Schopfer, V: Wellbrock, C.; Marais, R. Kature 2007, 445, 851.

5. Garbe, C.; Eigentler, T. K. Melanoma Res. 2007, 17, 117.

6. Koon, H. B.; Atkins, M. B. Expert Rev Anticancer Ther, 2007, 7.79

7. Wilhelm. S. M.: Carter, C.: Tang, L.; Wilkie, D.; Menabola, A.; Rong, H. Cancer Res. 2004, 64, 7099.

8. Strumberg, D.; Richly, H.; Hilger, R. A.; Schlencher, N.; Korfee, S. J. Clin. Oncol 2005, 23,965.

9. Clark, I. W.; Eder, J. P.: Ryan, D.: Lathia, C.: Lenz, H. J. Clin. Concer Res. 2005, 11,5472

10. Hirte, H. W: Moore, M: Siu, L; Oza, A: Hotte, S. T. Ann. Oncol. 2005, 16. 1688 .

11. Strumberg. D.: Voliotis, D.; Moller, J. G.; Hilger, R. A.; Richly, H.: Kredtke, S. Int. J. Chin. Phamacol. Ther 2002, $40,580$.

12. Richly, H.; Kupsh, P; Passage, K.; Grubert, M:; Voigtmanu, R:; Schwartz, B. Int. J. Clin. Phomtacol. Ther $2004,+2,650$.

13. Smith, R. A.; Barbosa, I.; Blum, C. L.; Bobko, M. A.: Caringal, Y. V.: Dally, R. Bioong. Wed Chem. Lett. 2001, 11, 2775.

14. Wood, J. E; Wild, H; Rogers, D. H.; Lyons, J.; Katz, M. E:; Caringal, Y. V. PCT Pat. Appl. WO 98052559, May 23, 1997.

15. Bankston, D.; Dumas, J.: Natero, R:; Riedl, D. R.: Monahan, M.-K. Org. Proc. Res. Dev. 2002, 6, 777.

16. Khire, U. R.; Bankston, D.; Barbosa, T.; Brittelli, D. R.; Caringal, Y.: Carlson, R. Bioorg. Med. Chent Leth. 2004, 14, 783.

17. Wan, P. T. C.: Gámett, M. T: Roe, S. M.: Lee, S.: Niculeseudunaz, D. Cell 2004, 116, 855 .

18. Eisen, T.: Ahmad, T.: Flaherty, K. T.: Gore, M: Kaye, S.; Marais, R.; Gibbens, I.; Hackett, S.; James, M.; Schuchter, L. M.; Nathanson, K. L.; Xia, C.; Simantov, R.; Schwartz, Bi; Poulin-Costello, M. O Dwyer, P. T.; Ratain, M. T. Br.J. Cancer 2006, 95,581 . 